\title{
Simplified design of the coulometric sensor-actuator system by the application of a time-dependent actuator current
}

\author{
W. Olthuis and P. Bergveld \\ MESA Research Institute, University of Twente, P.O. Box 217, 7500 AE Enschede (Netherlands)
}

\begin{abstract}
Acid or base concentrations can be determined by performing an acid-base titration with coulometrically generated $\mathrm{OH}^{-}$or $\mathrm{H}^{+}$ions at a noble metal actuator electrode in close proximity to the pH-sensitive gate of an ISFET. It is shown, both theoretically and experimentally, that the relation between the acid or base concentration and the time needed to reach the equivalence point in the resulting titration curve is linear in the case where the current through the actuator electrode varies with the square root of time. In addition, this square root of time-dependent actuator current results in an increased acid-to-base (and vice versa) transition speed at the equivalence point when compared to a constant actuator current pulse, enabling the equivalence point to be detected more accurately and easily.
\end{abstract}

\section{Introduction}

Acid or base concentrations can be determined by performing an acid-base titration with coulometrically generated $\mathrm{OH}^{-}$or $\mathrm{H}^{+}$ions at an actuator electrode in close proximity to a $\mathrm{pH}$ sensor. The titrant can be generated either by the electrolysis of water at a noble metal actuator electrode $[1,2]$ :

$2 \mathrm{H}_{2} \mathrm{O} \rightarrow 4 \mathrm{H}^{+}+4 e+\mathrm{O}_{2} \quad$ at the anode

and

$2 \mathrm{H}_{2} \mathrm{O}+2 e \rightarrow 2 \mathrm{OH}^{-}+\mathrm{H}_{2} \quad$ at the cathode

or by the reversible redox reaction of an iridium oxide actuator electrode, which is accompanied with the release or uptake of protons [3]:

$\mathrm{IrOOH} \rightleftharpoons \mathrm{IrO}_{2}+\mathrm{H}^{+}+e$

The $\mathrm{pH}$ sensor, which is used as an indicator electrode to detect the equivalence point in the titration curve, can consist of either a $\mathrm{pH}$-sensitive ISFET [1-3] or a $\mathrm{pH}$-sensitive iridium oxide electrode [4]. Figure 1 shows a schematic drawing of the first-mentioned device.

The equivalence time $t_{\mathrm{eq}}$, which is the time needed to reach the equivalence point in the titration curve, depends on the acid or base concentration of the sample solution. The coulometric sensor-actuator device optimally uses the fa- vourable properties of the $\mathrm{pH}$ sensor: its small, planar structure and, particularly for the ISFET, its small response time. Furthermore, the unfavourable long-term drift of the sensor does not cause any problems in this dynamic mode of measurement, because the sensor is only used as an indicator electrode and the absolute value of the output signal of the sensor is of no importance.

The titrant is generated by applying a current to the actuator electrode. The time dependence of this current determines, among other things, the relation between the acid or base concentration and the equivalence time, $t_{\mathrm{eq}}$. If the applied actuator current is a constant current pulse, then the square root of $t_{\text {eq }}$ is proportional to the acid or base concentration. However, if the actuator current varies with the square root of time, then $t_{\text {eq }}$

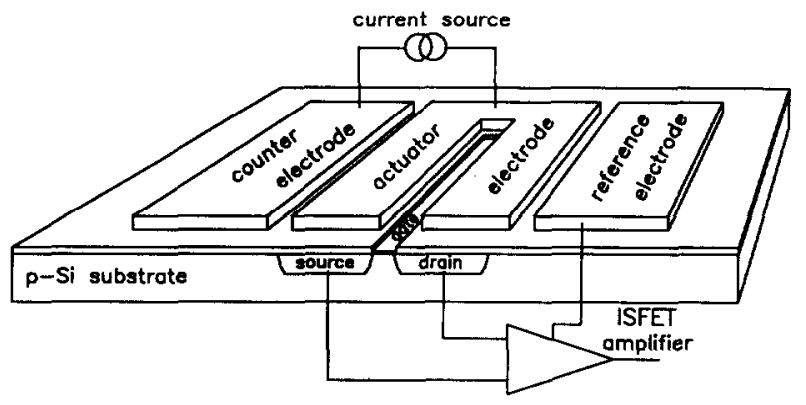

Fig, 1. The basic elements of an ISFET-based sensor-actuator system. 
is directly proportional to the acid or base concentration. This allows a more easy representation of the measurement results, which is desirable for future application of the device in a non-computerized portable set-up.

For this reason, and also to investigate to what extent the general theory of controlled current methods is applicable to the system, the relation between $t_{\mathrm{eq}}$ and the acid or base concentration as a function of the time-dependent actuator current is investigated and compared with corresponding measurement results.

\section{Controlled-current coulometry}

The dependence on the acid or base concentration of the time $t_{\mathrm{eq}}$ needed to reach the equivalence point in the titration curve is largely determined by the time needed to deplete totally the acid or base concentration at the surface of the actuator electrode, as depicted in Fig. 2.

In Fig. 2, the device is represented in one dimension, at which the actuator electrode is considered to be placed at $x=0$ and the sensor at $x=x_{\mathrm{s}}$. Two concentration profiles, occurring at the surface of the device during a coulometric titration, are shown: profile 1 shows the situation at the start of the titration in which the concentration is still homogeneous, whereas profile 2 represents the situation in which the original bulk concentration is depleted at $x=0$ by the ions generated at the actuator electrode. This moment (after the start of the ion generation) when the original bulk concentration at the actuator electrode surface virtually drops to zero is known as $t_{\text {Sand }}$ : $C\left(x=0, t=t_{\text {Sand }}\right)=0[5]$.

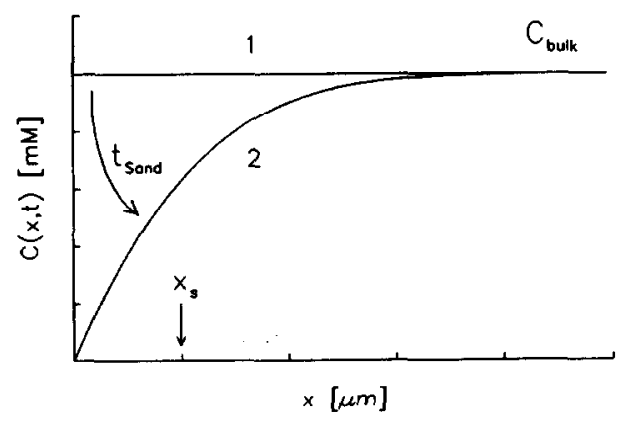

Fig. 2. Two concentration profiles occurring during a coulometric titration: curve 1 at the start of the titration and curve 2 at $t=t_{\text {Sand }}$. The actuator is placed at $x=0$ and the sensor at $x=x_{\mathrm{s}}$.
The sensor-to-actuator distance $x_{\mathrm{s}}$ is a complicating factor. However, especially for relatively large acid or base concentrations, the delay time, $t_{\text {delay }}$, caused by this distance $x_{\mathrm{s}}$ can be considered as a constant off-set [6].

If the applied actuator current is a constantcurrent pulse with current density $j_{\mathrm{c}}$, flowing through an acidic sample with bulk concentration

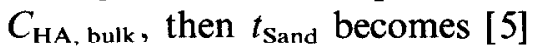

$t_{\text {Sand }}=\left\{\frac{C_{\mathrm{HA}, \text { bulk }} F\left(\pi D_{\mathrm{HA}}\right)^{1 / 2}}{2 j_{\mathrm{c}}}\right\}^{2}$

where $F$ is Faraday's constant $(96485 \mathrm{C} / \mathrm{mol})$ and $D_{\mathrm{HA}}$ is the diffusion coefficient of the titrated acid.

From eqn. (1) it is clear that the square root of $t_{\text {Sand }}$ is proportional to the bulk concentration $C_{\mathrm{HA}, \text { bulk }}$ to be determined. Because $t_{\text {eq }}=t_{\text {Sand }}+$ $t_{\text {delay }}$ and for relatively large $C_{\mathrm{HA} \text {, bulk }} t_{\mathrm{Sand}} \gg t_{\text {delay }}$, also $\left(t_{\mathrm{eq}}\right)^{1 / 2}$ is proportional to $C_{\mathrm{HA}}$, bulk .

For the reasons mentioned in the Introduction, it was decided to apply an actuator current $i_{\mathrm{c}}(t)$ to the electrode which varies with the square root of time [5]: $i_{\mathrm{c}}(t)=\beta t^{1 / 2}$. According to the theory, $t_{\text {Sand }}$ becomes in that case [5]

$t_{\text {Sand }}=\frac{2 C_{\mathrm{HA}, \text { bulk }} F A}{\beta}\left(\frac{D_{\mathrm{HA}}}{\pi}\right)^{1 / 2}$

where $A$ is the area of the actuator electrode.

In this equation, $t_{\text {Sand }}$, and thus $t_{\text {eq }}$, depends linearly on $C_{\mathrm{HA} \text {, bulk }}$. The generation of an actuator current that varies with the square root of time is possible with modern integrated circuits (e.g., with the AD534 [7]).

\section{Experimental}

\section{Preparation of the gold actuator/ISFET sensor device}

For the experiments described in this paper, a $\mathrm{pH}$-sensitive ISFET was used as the sensing device. The ISFET was fabricated following the standard NMOS processing steps, with an added tantalum oxide gate dielectric on top of the silicon oxide [8]. The generation of ions was performed at a gold actuator electrode, deposited around the gate. This thin gold film $(0.6 \mu \mathrm{m})$ was evaporated on a $\mathrm{Ti} / \mathrm{Ag}$ adhesion-promoting layer. The actuator electrode was patterned using standard photolithographic techniques. A layer of polyimide was spun on the wafer in which holes were etched both 


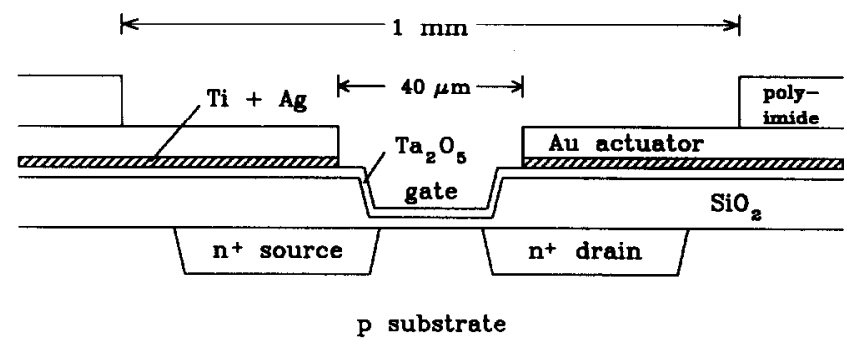

Fig. 3. Cross section of the $\mathrm{Ta}_{2} \mathrm{O}_{5}$-ISFET sensor with the gold actuator electrode.

to contact the ISFET and the actuator electrode and to free the area of the actuator electrode to be exposed to the solution. A cross section of the device is shown in Fig. 3. After dicing the wafer, the chips were glued on a $1 \mathrm{~cm} \times 5 \mathrm{~cm}$ piece of printed circuit board and connected to the available copper strips with bonding wires. The copper strips, the bonding wires and the edges of the chip were covered with epoxy for insulation and protection.

\section{Measurement set-up}

All measurements have been carried out with the set-up as shown in Fig. 4. The ISFET amplifier keeps both the drain current and the drain-source voltage constant at $100 \mu \mathrm{A}$ and $0.5 \mathrm{~V}$, respectively. The output voltage is denoted as $V_{\text {ISFET }}$ in the following Figures. All coulometric titrations were carried out in a $0.1 \mathrm{M} \mathrm{KNO}_{3}$ supporting electrolyte at room temperature. Both the constant actuator electrode current and the current varying with the square root of time were generated by a computer-controlled current source. The time $t_{\mathrm{eq}}$ was determined by the position of the extreme of the first derivative of the resulting titration curve.

\section{Results and discussion}

\section{Acid-to-base transition speed}

The results of two typical acid-base titrations in a relatively low concentration of $\mathrm{HNO}_{3}$ $\left(C_{\mathrm{HA} \text {, bulk }}=2 \mathrm{mM}\right)$, carried out with one and the same device, are shown in Fig. 5.

In the experiment of Fig. 5(a), the actuator current was a constant-current pulse, $j_{\mathrm{c}}=$ $20 \mu \mathrm{A} / \mathrm{mm}^{2}$, and in that of Fig. 5(b), the current increased with the square root of time, $\beta=$

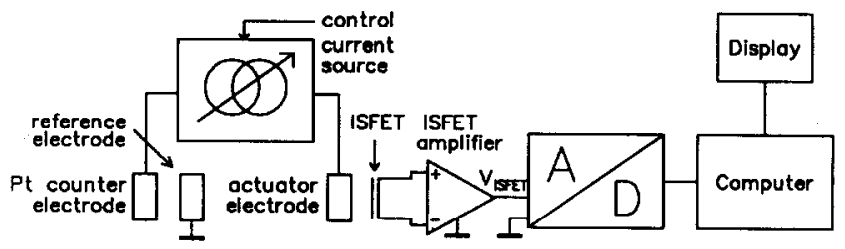

Fig. 4. Measurement set-up.

$22.5 \mu \mathrm{A} / \mathrm{s}^{1 / 2}$. Both curves show a $\Delta V_{\text {ISFET }}$ of $\approx 0.45 \mathrm{~V}$, corresponding to a $\Delta \mathrm{pH}$ of about 8 . (The pH sensitivity of a $\mathrm{Ta}_{2} \mathrm{O}_{5}$-ISFET is approximately $-55 \mathrm{mV} / \mathrm{pH}$.) The steeper the titration curve around the equivalence point, the more accurate the detection of $t_{\mathrm{eq}}$ is.

Therefore, it is interesting to compare the acidto-base transition speed, $v_{\mathrm{tr}}$, at the equivalence point. The measurement results give $v_{\text {tr }}$ (constant current) $=46 \mathrm{pH} / \mathrm{s} \quad$ (Fig. $5(\mathrm{a}))$ and $v_{\mathrm{tr}}$ (square root current) $=68 \mathrm{pH} / \mathrm{s}$ (Fig. $5(\mathrm{~b})$ ). It is not realistic to compare these measured values with the values that can be derived from eqns. (1)

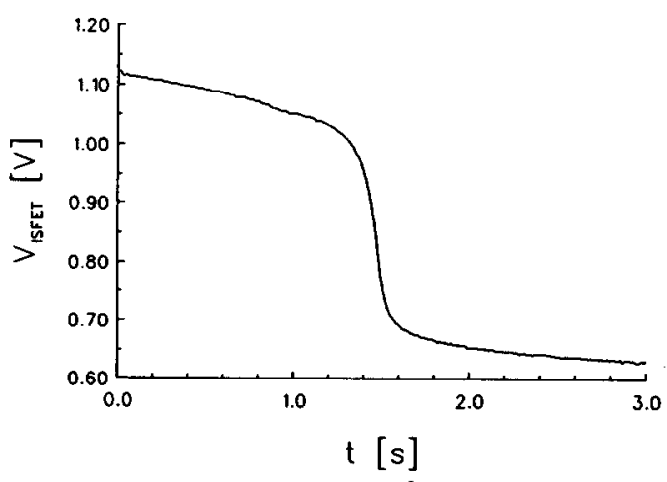

(a)

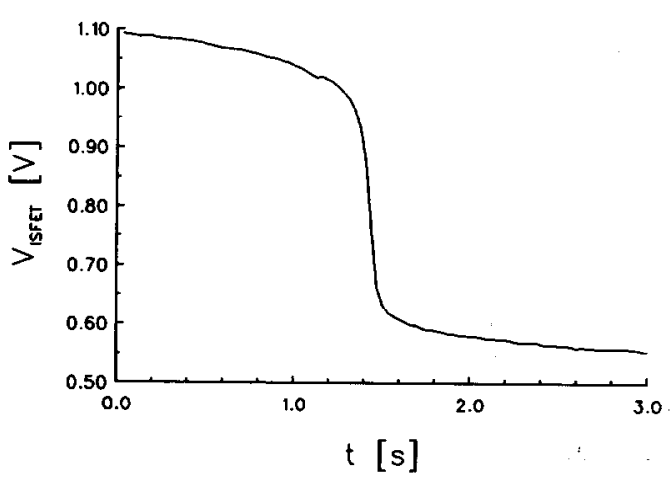

(b)

Fig. 5. Two typical acid-base titrations in $2 \mathrm{mM} \mathrm{HNO}_{3}$ : (a) with a constant actuator current pulse, $j_{\mathrm{c}}=20 \mu \mathrm{A} / \mathrm{mm}^{2}$ and (b) with an actuator current increasing with the square root of time, $\beta=22.5 \mu \mathrm{A} / \mathrm{s}^{1 / 2}$. 
and (2), because these equations describe the profiles at the surface of the actuator electrode, at $x=0$, whereas the sensor measures at $x=x_{\mathrm{s}}$, where the concentration gradient is decreased compared to the gradient at $x=0$. However, it is possible to find an expression for the ratio of the acid-to-base transition speed, $v_{\mathrm{tr}}$, for the two different actuator currents. The ratio of the two transition speeds is obtained by calculating the quotient of $\partial C_{\mathrm{HA}, \text { bulk }} / \partial t$ from eqns. (1) and (2) at $t=t_{\text {Sand }}$ :

$\frac{v_{\mathrm{tr}}(\text { square root current })}{v_{\mathrm{tr}}(\text { constant current })}=\frac{\pi \beta F C_{\mathrm{HA}, \text { bulk }}\left(\pi D_{\mathrm{HA}}\right)^{1 / 2}}{4 j_{\mathrm{c}}^{2} A}$

By substitution of the values given at the beginning of this Section and with $D_{\mathrm{HA}}=9.3 \times 10^{-9} \mathrm{~m}^{2} / \mathrm{s}$ and $A=1 \mathrm{~mm}^{2}$, the calculated ratio is 1.46 . The ratio found by the results of the measurements is $1.48(=68 / 46)$, which means a good agreement.

It can be concluded that under comparable experimental conditions, the acid-to-base transition speed for the titration with the square root actuator current is higher than that for the titration with a constant-current pulse. As a result of this, $t_{\text {eq }}$ can be detected with a higher accuracy. Moreover, because the equivalence point is detected by the extreme of the first derivative, this can be done with a larger signal-to-noise ratio.

\section{Acid and base concentration determination}

The results of measurements performed with a constant-current pulse in acidic as well as in alkaline samples are reported elsewhere [1-4] and show the expected linear relation between the square root of $t_{\text {eq }}$ and the acid or base concentration, with a slope in accordance with eqn. (1).

The results of measurements in acetic acid (HAc) and in $\mathrm{KOH}$, carried out with an actuator current depending on the square root of the time, are shown in Fig. 6. At each concentration, the measurement was repeated several times in order to check the reproducibility of the experiments. The results at a fixed concentration never varied more than $2 \%$. After each measurement, there was a pause of about one minute before the next measurement was started to avoid erroneous results due to convection.

As expected from eqn. (2), the relation between the concentration and the time needed to reach the

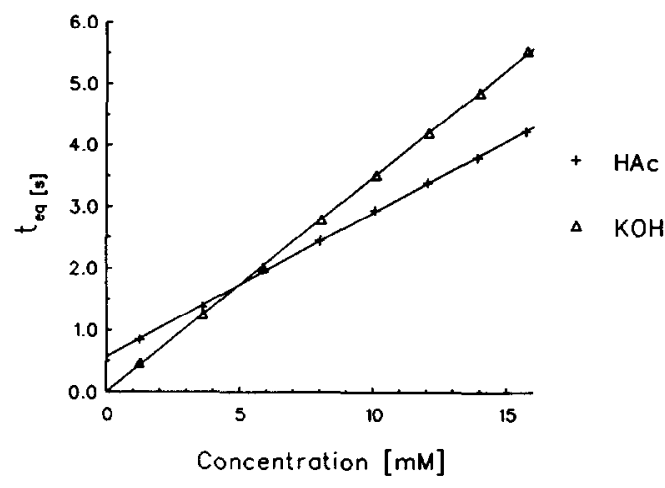

Fig. 6. The time $t_{\text {eq }}$ needed to reach the equivalence point as a function of the $\mathrm{HAc}$ and $\mathrm{KOH}$ bulk concentrations, using an actuator current increasing with the square root of time.

equivalence point is now linear: the correlation coefficient of the linear line fitted through the measured data is $>0.9997$ for both curves of Fig. 6.

The slope of the linear line through the measurement data of $\mathrm{KOH}$ is $0.348 \mathrm{~s} / \mathrm{mM}$, and that expected from eqn. (2) is $0.351 \mathrm{~s} / \mathrm{mM}$. The agreement between measurement results and theory in the case of the HAc measurement is less favourable: a slope of $0.168 \mathrm{~s} / \mathrm{mM}$ is expected, whereas a slope of $0.233 \mathrm{~s} / \mathrm{mM}$ is measured. This repeatedly found deviation is probably caused by the choice of the diffusion coefficient $D_{\mathrm{HA}}$ in eqn. (2) for calculating the theoretical slope. For the calculations, the relatively small $D_{\mathrm{HA}}$ for undissociated HAc molecules is used, whereas it is more realistic to use a mean diffusion coefficient composed of both the HAc molecules and the relatively fast $\mathrm{H}^{+}$ions, which would result in an increased calculated slope.

The sensor-to-actuator distance introduces a delay time, causing a non-zero $t_{\mathrm{eq}}$ in the absence of any acid or base in the bulk solution. There is, however, no analytical expression for this delay time in the case where a square root actuator current is used. The difference in off-set between the HAc and the $\mathrm{KOH}$ curves as observable in Fig. 6 might be due to dissolved carbon dioxide, causing an initial acidification of the sample solution, resulting in the observed shift of the curve.

It can be concluded that the relation between the acid or base concentration and the measured time $t_{\text {eq }}$ needed to reach the equivalence point is linear when an actuator current is used that depends on the square root of time. This linear relation is expected from the theory and enables a 
simplified design coulometric sensor-actuator system to be achieved.

\section{References}

I W. Olthuis, B. H. van der Schoot, F. Chavez and P. Bergveld, A dipstick sensor for coulometric acid/base titrations, Sensors and Actuators, 17 ( 1989) 279-283.

2 W. Olthuis, J. Luo, B. H. van der Schoot, J. G. Bomer and P. Bergveld, Dynamic behaviour of ISFET-based sensor-actuator systems, Sensors and Actuators, BI (1990) 416-420.

3 W. Olthuis, J. G. Bomer, P. Bergveld, M. Bos and W. E. van der Linden, Iridium oxide as actuator material for the ISFET-based sensor-actuator system, Sensors and Actuators B, 5 (1991) 47-52.
4 W. Olthuis, J. C. van Kerkhof, P. Bergveld, M. Bos and W. E. van der Linden, Preparation of iridium oxide and its application in sensor-actuator systems, Sensors and Actuators B, 4 (1991) 151156.

5 A. J. Bard and L. R. Faulkner, Electrochemical Methods, Fundamentals and Applications, Wiley, New York, 1980, pp. 253-256.

6 W. Olthuis, J. Luo, B. H. van der Schoot, P. Bergveld, M. Bos and W. E. van der Linden, Modelling of non-steady-state concentration profiles at ISFET-based coulometric sensor-actuator systems, Anal. Chim. Acta, 229 (1990) 71-81.

7 Analog Devices, 1988 Linear Products Databook, Analog Devices, Inc., 1988, pp. 6-13.

8 P. Bergveld and A. Sibbald, Analytical and biomedical applications of ion-selective field-effect transistors, in G. Svehla (ed.), Comprehensive Analytical Chemistry, Vol. XXIII, Elsevier, Amsterdam, 1988. 